\title{
El reciclaje de vidrio y sus beneficios en el medio ambiente.
}

\section{The recycling of glass and its benefits in the environment.}

\author{
Dr. Tatiana Carrasco R. ${ }^{1}$
}

\section{Resumen.}

El reciclaje es la mejor forma para ayudar al medio ambiente, el hombre se ha convertido en el causante de contaminar día a día el medio en que vivimos, sin darnos cuenta hemos venido matando nuestra naturaleza cada vez más, ya es hora que tomemos un poco de conciencia y ayudar en algo para recobrar su ambiente, es por ello que esta investigación se hablara sobre el reciclaje del vidrio y como su reusó ayudara al medio ambiente y disminuir un poco la contaminación que se da con el desecho del vidrio, el reciclaje del vidrio es uno de los más gratificante ya que se puede reciclar al 100\% sin desperdiciar nada, incluso existen envases de vidrio que con solo una buena limpieza queda como nuevo y permite volverlo utilizar ya sea en su misma función para la que fue elaborada o en otra que se estime conveniente. El reciclaje del vidrio beneficia tanto al ser humano como al medio ambiente, al ser humano le permite por medio del reciclaje obtener incluso hasta ingresos económicos y al medio ambiente ayudamos a no contaminarlo más, para poder crear conciencia en el ser humano es muy importante hablar del medio que sus y como aporta a la vida del ser humano es por ello que en la investigación se trataran puntos muy importantes sobre lo que es el reciclaje, medio ambiente y el vidrio, además se tratara de hacer ver el aporte que esta da a la naturaleza ,principalmente, el reciclaje de vidrio. Según las estadísticas encontradas Europa es el continente que más recicla en el mundo, España se ha convertido en el país con la tasa de reciclaje más alta, en América Latina el reciclaje aun no es un tema de éxito y mucho menos en nuestro país.

Palabras claves: Beneficios, reciclaje, medio ambiente, cristalización.

\begin{abstract}
.
Recycling is the best way to help the environment, man has become the cause of pollute every day the environment in which we live, without realizing we have been killing our nature more and more, it is time we take a little of conscience and help in something to recover their environment, that is why this research will talk about the recycling of glass
\end{abstract}

${ }^{1}$ Visionario digital, Ambato,Ecuador,tatianacarrasco@cienciadigital.org 
and how it reused it will help the environment and reduce a bit the pollution that occurs with the waste of glass, the recycling of glass is one of the most rewarding since it can be recycled $100 \%$ without wasting anything, there are even glass containers that with just a good cleaning remains as new and allows it to be used again in its same function for which it was made or in another that is considered convenient. The recycling of glass benefits both the human being and the environment, the human being allows through recycling to obtain even economic income and help the environment not to contaminate it more, to be able to create awareness in the human being it is very important to talk of the medium that their and as it contributes to the life of the human being is for it that in the investigation they will be treated very important points about what is the recycling, environment and the glass, in addition it will try to make see the contribution that this gives to nature, mainly, the recycling of glass. According to the statistics found, Europe is the continent that recycles the most in the world, Spain has become the country with the highest recycling rate, in Latin America recycling is not yet an issue of success, much less in our country.

Keywords: Impact, technological advances, initial education.

\section{Introducción}

\section{El vidrio}

\section{Según los autores (Isam A.2013, Claudio P.2015, Aguilera N.2015, Schmelzer J.2016, Tórrense R.2015, Mata A.2014)}

El vidrio es una mezcla de varias sustancias que no tiene composición constante; ya que varía según el tipo de vidrio. No obstante, está formado principalmente por sílice, He aquí las principales materias primas y el porcentaje aproximado en que cada una entra en la composición del vidrio, fue muy utilizado en las vidrierías de las cátedras goticas.La vidrio admite un reciclaje total, y ello significa que se trata de un material ideal para ser reciclado al completo .Además podemos hacerlo tantas veces como queramos sin modificarlo. La industria que se dedicaba a la fabricación del vidrio experimento un gran cambio a partir del primer cuarto siglo XIX. Comenzaron a fabricarse vidrios de alta calidad para ser utilizados en instrumentos ópticos. Se explora la relevancia de los conceptos de fragilidad y dela temperatura de transición vítrea para la compresión de la nucleación cristalina y el crecimiento en líquidos formadores de vidrio .Se muestra que la fragilidad clásica puede ser relevante para la comprensión del comportamiento de cristalización solo si se cumplen varias condiciones. Una característica clave que distingue a la fabricación de vidrio de la fabricación de metal es la ausencia de escoria duradera que ayudaría a localizar los sitios de fabricación de vidrio, y proporcionar pistas sobre las materias primas y las condiciones de fusión. Estos se eliminaron de una u otra durante el proceso y debido a su solubilidad en el agua casi inmediatamente también se perdieron del registro arqueológico. El primer paso en el proceso de reciclado de vidrio es la limpieza. Aunque el vidrio se encuentre mezclado en distintos colores, no influye para la producción de nuevos envases, ya que al vidrio de color, se le trata con decolorante. 
Figura 1: El Vidrio

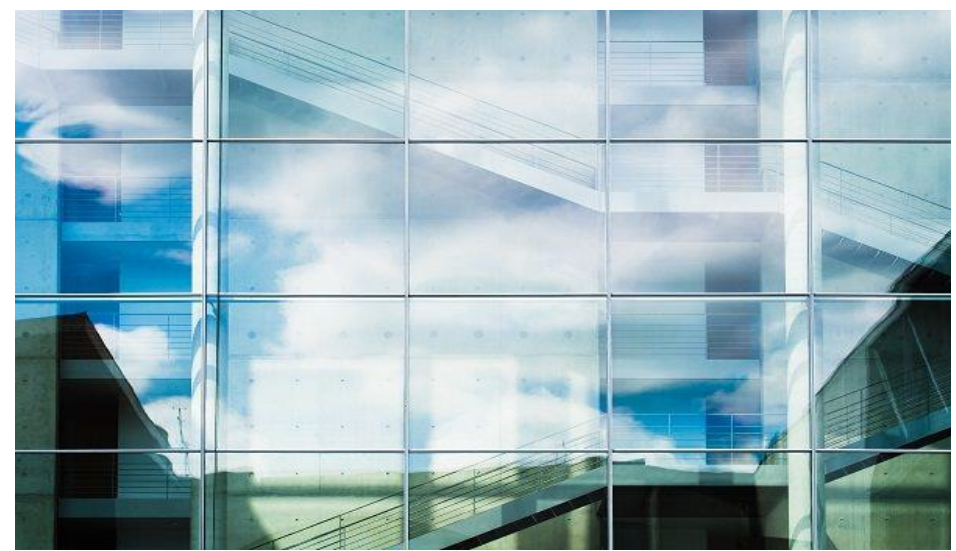

Fuente: httphttphttps://cienciatoday.com/fabrica-vidrio-tipos/

Autor: Elena Verger

El vidrio es un vasto mineral quebradizo que se puede reciclar en su totalidad, está compuesto de varias sustancias no siempre las mismas ya que varía dependiendo de su variedad pero en la mayoría contiene sílice. En el siglo XVIII, comenzaron a elaborar cristales de alta eficacia que se comenzaron a convertir en instrumentos ópticos siempre reflejando la fragilidad en sus productos.

Es un material cristalizado que se ha venido convirtiendo en un objeto muy utilizado en la vida diaria del ser humano, gracia a su elegancia que brinda hace que su demanda crezca cada día más, desde las décadas antiguas ha venido innovando en diferentes colores modelos y estilos haciendo crecer su mercado a nivel mundial.

El vidrio es material de sílice que gracias al calor de un horno se le da las diferentes formas de objetos ,tales como vasos, botella, laminas para ventanas .puertas vitrinas y demás obras en las que es utilizado, es por ello que se afirma que el vidrió brinda elegancia en la vida del ser humano. Los objetos creados de este material llamado vidrio tienen un lugar muy importante en diferentes lugares del mundo es decir los objetos creados de este material gracias a su elegancia su demanda de obtenerlos crece día tras día se dice que cada persona al menos tiene un objeto que sea de vidrio ya sea un adorno un frasco de perfume o porque no una bebida en su casa o departamento desde la llegada del vidrio que fue antes de Cristo ha venido innovando tanto en la fragilidad como en su color cada las industrias sacan diferentes envases que nos asombran con su presencia y elegancia, un vaso de vidrio en una mesa es la pieza fundamental, el obtener un objeto de vidrio se ha convertido en una necesidad humana, no por ser frágil disminuyo su consumo sino que a lo contrario es su fragilidad lo que le da la elegancia necesaria para convertirse en un ente de mucha utilidad.

\section{Medio ambiente.}

Según los autores (Melanv V.2014, Martínez M.2014, Barr S. 2016, Islam A.2016, Basterra N.2014, Medina M.2016). 
El medio ambiente es el espacio en el que se desarrolló la vida de los seres vivos y permite la interacción de los mismos .Sin embargo este sistema no solamente está conformado por seres vivos, sino que también por elementos abióticos y por elementos artificiales.

Siempre ha sido un tema importante para el desarrollo y sostenibilidad de la calidad de vida de los seres vivos pero si hacemos un análisis detallado del asunto nos podemos dar cuenta que desde el siglo XXI. El medio ambiente y la sociedad exploran formas de promover los cambios de comportamientos necesarios para crear una sociedad sostenible. A través de un enfoque critico de los vínculos entre sostenibilidad, política y participación ciudadana. Tal integración de los estilos de vida sostenibles probablemente sea el único medio eficaz para involucrar a la mayoría de los ciudadanos en el debate ambiental, dado la gran influencia de la sociedad de consumo aspiraciones y creencias individuales. Millones de toneladas de vidrio residual se generan anualmente en todo el mundo .Una vez que el vidrio se convierte en un residuo, se desecha como vertederos. Lo cual es insostenible ya que esto se descompone en el ambiente .El vidrio está compuesto principalmente de silice.Un uso de molido el desecho de vidrio en el concreto como remplazo parcial del cemento podría ser un paso importante hacia el desarrollo ambientalmente, En los primeros años del siglo XIX, se otorgaba al ambiente una connotación geográfica, fisca fue Charles Darwin quien supera esta dimensión del concepto aportando su visión orgánica y enfatizando la importancia de estudiar las interrelaciones entre los ecosistemas. Como unidad funcional los estudios son abordados por otra rama de la ecología que es la sinecología que estudia la dinámica del ecosistema es su conjunto, en sus procesos reguladores.

Figura 2: Medio Ambiente

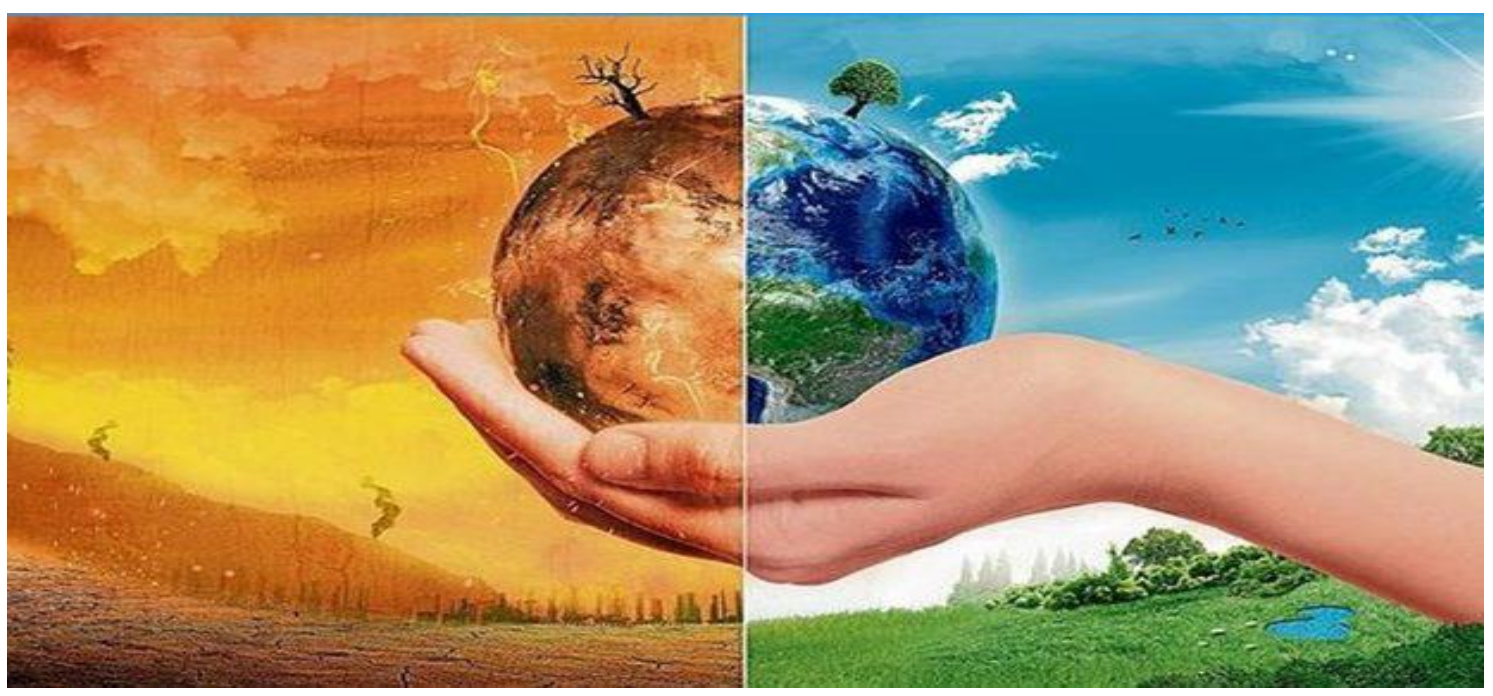

Fuente:httphttp://www.resumenlatinoamericano.org/2017/05/18/medioambiente -e-igualdad-social-por-alvaro-garcia-linera/

Autor: Álvaro García

El medio ambiente es el pasaje en el que se despliega la vida de los seres vivos siempre ha sido un tema muy importante para el desarrollo y sostenibilidad, la sociedad explora forma de promover los cambios necesarios para crear una sociedad sostenible. Es el medio mediante en el cual se desarrolla la vida del ser humano, este tema se ha convertido 
en un lema muy común en discursos de políticos en sus campañas confirmando en ayudar a reducir la contaminación que afecta día a día su ecosistema, la contaminación ambiental afecta no solo la vida de todo ser vivo debemos de poner un poco de parte nuestra en ayudar a reducir esta contaminación a nuestra casa, el ser humano se ha convertido en la principal fuente de contaminación ambiental.

Al hablar de medio ambiente estamos haciendo énfasis en todo lo que nos rodea desde un objeto hasta un ser que tiene vida, es el medio en el cual se desarrolla un ser vivo, por ello es muy importante ayudarlo a conservar su naturaleza ya que esta permite dar el oxígeno que el ser vivo necesita a diario ,si no cuidamos nuestro ambiente que es lo nos rodea quien lo hará, una pregunta que debemos hacernos y nosotros mismos responderla, para así llegar a hacer conciencia y dejar de destruirlo, si todos se dedicaran a sembrar un árbol en vez de talarlo y de recoger toda basura que vieran en las calles ayudarían muchísimo a conservar en medio ambiente ya si se pone un granito de arena como se dice comúnmente serviría de mucha ayuda y no tendríamos arrepentimiento en el futuro si no que al contrario nos sentiríamos satisfecho de haber hecho algo por la naturaleza que nos rodea.

\section{Reciclaje de vidrio}

Según los Autores (Vigil J.2018, Leonardo 2017, María R. 2014, Gómez A. 2017, Guerrero L.2018, Arias P.2014, Bidegaray I.2018, R del Valle .2017, Cano J.2015, Vikinga. 2014)

El vidrio es el único material que puede ser recuperado en su totalidad. La cultura y la economía de los residuos tienen expresión en este tipo de material formado fundamentalmente por sílices y otras bases. Reciclar vidrio es una buena forma de colaborar en la protección del medio ambiente global, de cada kilogramo de envase de vidrio reciclado se obtiene un kilogramo de nuevos envase. La regla de las tres $\mathrm{R}$ de la ecología tiene su origen en una organización no gubernamental Greenpeace y que Japón defendió durante una cumbre del G8 en 2004, esta idea promueve tres pasos básicos para disminuir la producción de residuos y así contribuir a la protección y conservación del medio ambiente. Al apostar por Reducir, Reutilizar Y Reciclar (las 3 R) se trata de cambiar los hábitos de consumo de la sociedad, haciéndolos más responsables y sostenibles. Cuando hablamos de reducir lo que estamos diciendo es que debe tratar de reducir o simplificar el consumo de los productos directos, o sea, todo aquello que se compra y se consume, ya que esto tiene una relación directa con los desperdicios, y a la vez con nuestro bolsillo. Reutilizar es dar un nuevo uso a un material u objeto. Este nuevo uso puede ser el mismo para el cual fue fabricado o puede ser diferente. Reciclar es la $\mathrm{R}$ más común y menos eficaz. Se trata de rescatar lo posible de un material que ya no sirve para nada (comúnmente llamado basura) y convertirlo en un producto nuevo. Por ejemplo, una caja vieja de cartón se puede triturar y a través de un proceso industrial o casero convierte a papel nuevo. Lo bueno del reciclaje es que actualmente se puede reciclar casi todo tipo de basura y muchos municipios ya tienen los servicios de reciclaje integrados a su sistema de recolección de basura.

Datos estadísticos del reciclaje a nivel mundial. El 73\% del vidrio de Europa se recicla España registra una tasa del $70 \%$ superando a grandes potencias como Reino Unido, por primera vez la Europa de los 28 alcanza una tasa de reciclaje del 73\%, así lo revelan las 
últimas cifras publicadas por la federación Europea de envases de vidrio (FEVE).Con más de 25 mil millones de envases de vidrio reciclados, la industria se mantiene como uno de los mejores ejemplos de economía circular.

Dinamarca, Suecia, Bélgica, Luxemburgo, Austria y Alemania encabezan el listado de países con la mayor tasa de reciclaje de Europa, superando el 90\%en todos los casos y alcanzando hasta un $98 \%$ en Dinamarca. Por su parte Italia (76\%), países bajos (79\%) y Malta (21\%) han registrado un mayor crecimiento respecto a años anteriores, Europa se ha convertido en el continente con las mayores tasas de reciclaje del mundo. (Cano, 2015)

En América Latina el tema del reciclaje está en pañales. México produce hasta 95.000 toneladas de basura al día reciclando solo un 17.3\%.Le sigue Ecuador con 16,8\%.Venezuela con un 15\%.Colombia 14\%, Argentina 11\%, Chile 10\%, Bolivia 2,8\%, y Perú 2\%.esto según aproximaciones y datos de las ONG'S y Organizaciones Gubernamentales entre 2011 y 2012. (Vikinga, 2014)

Datos estadísticos del reciclaje en ecuador a nivel nacional, entre los años 2010 y 2014, se incrementó el porcentaje de hogares que clasificaron residuos inorgánicos. Cabe aclarar que recuperar materiales reciclables disminuye la cantidad de residuos sólidos que se depositan en los sistemas de relleno sanitario, y se prolonga la vida útil de estos residuos. Al disminuir el volumen de los residuos sólidos destinados a relleno sanitario, los costos de recolección y disposición final son enormes .El uso de materiales reciclables como materia prima en la manufactura de nuevos productos ayuda a conservar recursos naturales renovables y no renovables. Dentro de los distintos residuos clasificados en los hogares en el año 2014, el plástico fue el residuo con mayor clasificación (31,48\%), en comparación al papel-cartón $(20,86 \%)$ y el vidrio $(12,68)$.

Figura 3: Reciclaje de Vidrio.

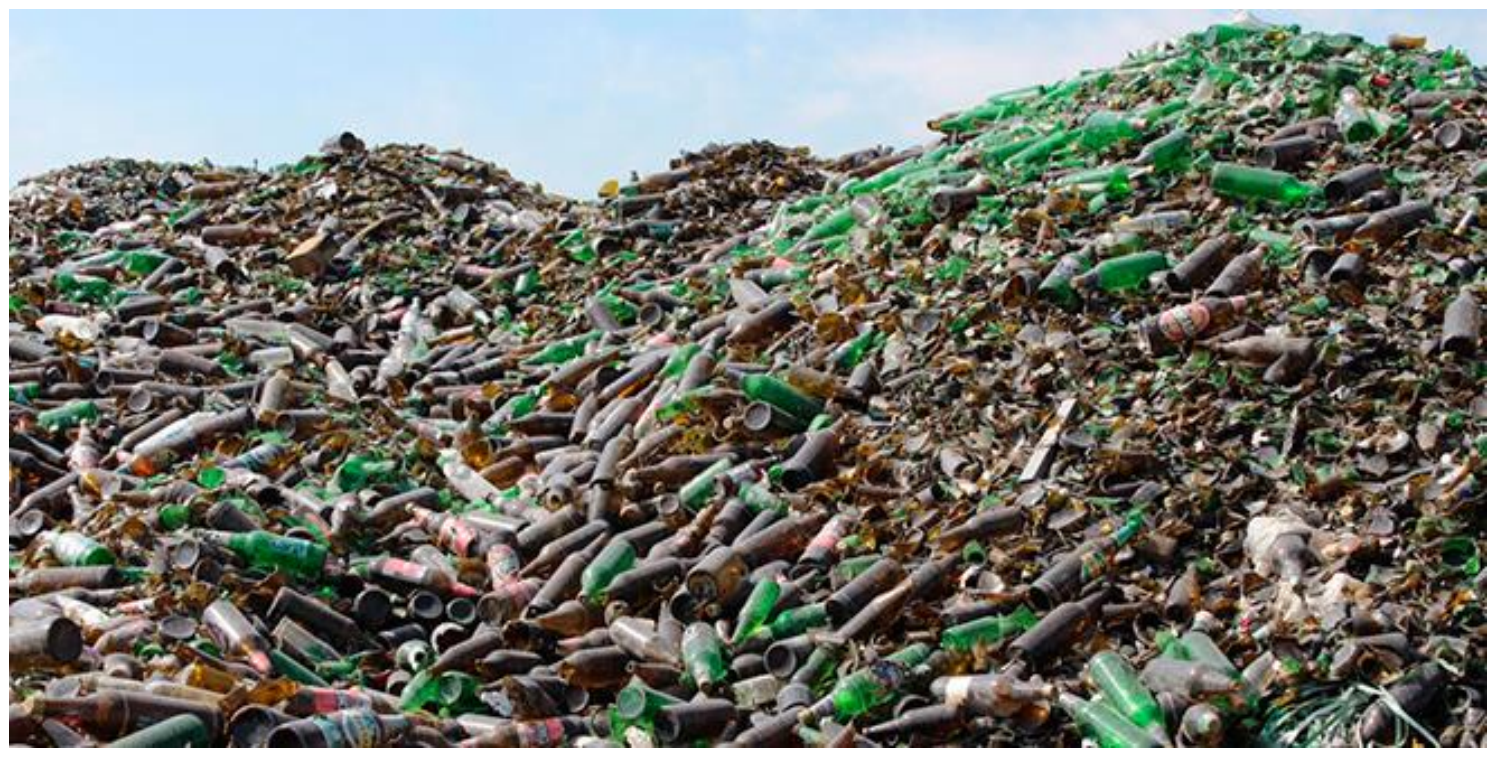

Fuente: https://sustempo.com/reciclaje-vidrio-chile-cristal-esperanza

Autor: Cristina González 
Figura 4: Datos del reciclaje en Ecuador

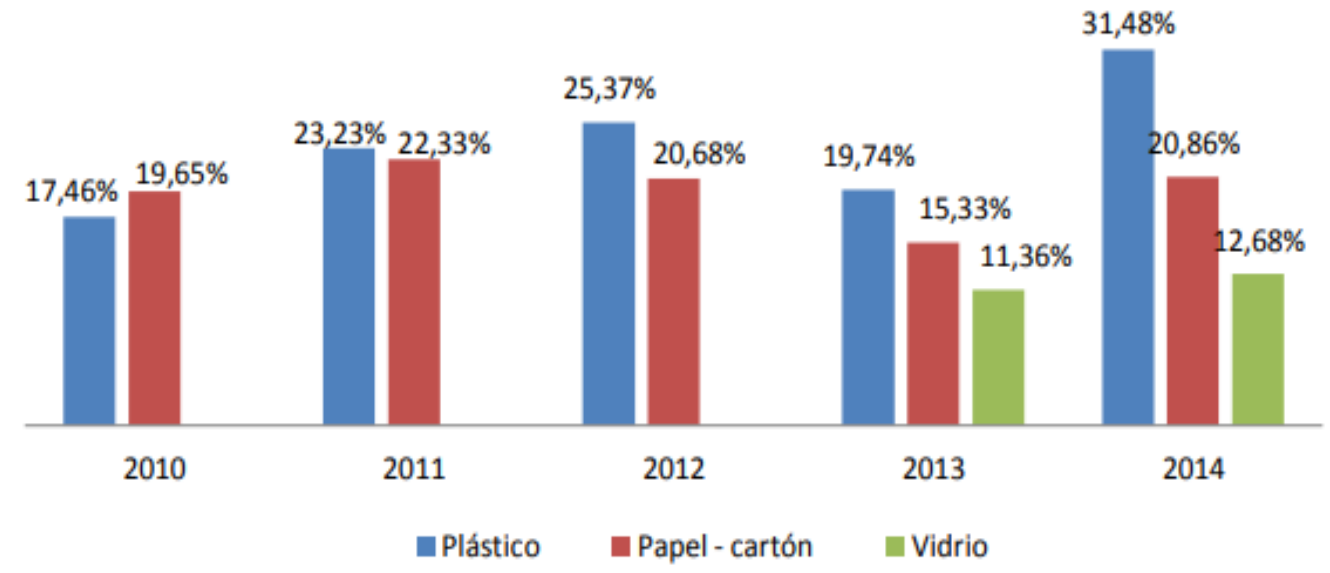

Fuente:http://www.ecuadorencifras.gob.ec/documentos/web-

inec/Encuestas_Ambientales/Hogares_2014/Documento_tecnico_Modulo_Ambi ental_Hogares_2014.pdf

Autor: Pamela Arias \& Marine Seilles

El reciclaje del vidrio se da es su totalidad ya que este permite reciclarse al 100\%, este permite realizar actividades económicas, en los últimos años el reciclaje se ha convertido en el principal ingreso económico de muchos hogares a nivel mundial, el reciclaje no solo ayuda económicamente a muchas familias sino que también aporta muy grandemente a nuestro ecosistema ya que se disminuye la contaminación que producen los desechos.

En el ecuador el reciclaje de vidrio es el más bajo según el censo de estadística del INEC, en el año 2013-2014 fue entre el 11\% y 13\% lo cual indica que existe un bajo reciclado de vidrio, por lo hay que trabajar más en concientizar a la gente en el reciclaje de vidrio, ya que este ayuda a disminuir la contaminación que este produce, es para ello que debemos ser más consiente con la basura que se vota diariamente, y tratar de dar otro uso a las cosas ya que así será una manera de ayudar nuestro planeta. Los desechos arrojados a diario son los principales causantes de contaminación a la naturaleza, aplicando la regla de las tres RRR, ya que su aplicación es de gran utilidad para el medio ambiente.

Según las estadísticas encontradas Europa es el continente con mayor reciclaje a nivel mundial, España el país que más recicla desechos, mientras que en América Latina en él te del reciclaje aún falta mucho por hacer ya que no se ha convertido en costumbre lo del reciclaje, es decir falta mucho por hacer por nuestro planeta si todos países reciclaran como España la contaminación ambiental disminuyera en un alto porcentaje, pero no hay que darse por vencidos según mi criterio si los gobiernos ayudaran un poco seria más fácil ya que con la ayuda de ellos como autoridades este tema seria de más importancia y podríamos así llegar hacer una sola fuerza para disminuir la contaminación que la basura causa a diario al planeta, de esta forma se demuestra un poco en esta investigación las estadísticas de reciclaje a nivel internacional y nacional, como forma de demostrar lo poco o mucho que se está haciendo para mejorar el ecosistema y el medio ambiente ya que si no hacemos conciencia hoy nos arrepentiremos en un futuro ya que será tarde ,aún 
se está a tiempo de hacer conciencia y mejor en el tema del reciclaje, ya que reciclando es la mejor manera de ayudar a nuestro planeta tierra.

El reciclaje de vidrio es el que más poco se da según las estadísticas encontradas a pesar de que este material es cien por ciento reciclable la sociedad aun no ase conciencia y prefiere darle un solo uso se debería coger como ejemplo España un país que no solo es el pionero del reciclaje sino que también el primero y con las estadísticas más altas en el reciclaje de vidrio ellos le dan diferente reusó a este material, los países latino deberían de coger esta costumbre del reciclaje de vidrio, ya que además de convertirse en una fuente de ingreso para los hogares también se ayuda conservar el medio ambiente, como beneficio del reciclaje de vidrio se obtendría la disminución del consumo de esta materia prima ,ayuda a la naturaleza, se convierte en una fuente laboral de muchos hogares y muchos beneficios más que esto desencadena.

\section{Conclusiones}

$>$ El vidrio es un material que a pesar de los años no pierde su elegancia gracias a su cristalización.

$>$ Medio ambiente es el contorno en que se desarrolla la vida del ser vivo, es por ello que mediante el reciclaje se reducen los altos índices de contaminación.

$>$ Reciclar vidrio evita la extracción de nueva materia prima y ayuda a disminuir la gran cantidad de desechos de este material.

$>$ Según las estadísticas encontradas Europa es el Continente con la mayor tasa de reciclaje.

\section{Referencias Bibliográficas}

Aguilera, N. (2015). EL VIDRIO. Obtenido de https://es.slideshare.net/nileydisaguilera/el-vidrio-44410560?qid=1d1515d62f7a-4417-883d-629b3253b688\&v=\&b=\&from_search $=5$

Arias, P., \& Marine Sailles. (2014). Informacion Ambiental en Hogares. Obtenido de http://www.ecuadorencifras.gob.ec/documentos/webinec/Encuestas_Ambientales/Hogares_2014/Documento_tecnico_Modulo_Ambi ental_Hogares_2014.pdf

Barr, S. (2016). Environment and Society. Obtenido de https://www.taylorfrancis.com/books/9781317142393

Basterra, N., \& Peralta, E. (2014). INTRODUCCIÓN A LA EDUCACIÓN. Obtenido de http://cegae.unne.edu.ar/docs/IntrodALaEduacionAmbiental.pdf

Bidegaray, I. (2018). Tesserae Recycling in the Production of Medieval Blue Window Glass. Obtenido de https://onlinelibrary.wiley.com/doi/abs/10.1111/arcm.12350 
Cano, J. (2015). El 73\% del vidrio de Europa se recicla. Obtenido de https://www.ecoticias.com/residuos-reciclaje/106996/vidrio-Europa-recicla

Claudio, P. (2015). HISTORIA DEL VIDRIO TIPOS Y TÉCNICAS DE FABRICACIÓN ORIGEN. Obtenido de https://historiaybiografias.com/historia_vidrio/

Gómez, A. (2017). Medio Ambiente. Obtenido de http://www.telam.com.ar/notas/201706/193121-reciclar-reutilizar-reducirbasura-medio-ambiente.html

Guerrero, L. (2018). Cómo seguir la regla ecológica más importante: definición de las 3R. Obtenido de https://www.aboutespanol.com/las-tres-erres-ecologicasreducir-reutilizar-reciclar-3417851

http://www.telam.com.ar/notas/201706/193121-reciclar-reutilizar-reducir-basuramedio-ambiente.html. (2017). http://www.telam.com.ar/notas/201706/193121reciclar-reutilizar-reducir-basura-medio-ambiente.html. Obtenido de http://www.telam.com.ar/notas/201706/193121-reciclar-reutilizar-reducirbasura-medio-ambiente.html

Isan, A. (2013). Ventajas del reciclaje de vidrio. Obtenido de https://ecologismos.com/ventajas-del-reciclaje-de-vidrio/

Islam, S. (2016). Waste glass powder as partial replacement of cement for. Obtenido de https://ac.els-cdn.com/S2212609016301157/1-s2.0-S2212609016301157main.pdf?_tid=75b66683-c328-4974-892ff7643eb35192\&acdnat=1539656532_6561718726a72e116255f6c0126512ef

Leonardo. (2017). Las tres $\mathrm{R}$ de la ecología: reducir, reutilizar y reciclar. Obtenido de https://www.leonardo-gr.com/es/blog/tres-r-ecologia-reducir-reutilizar-reciclar

Maria, R. (2014). Regla de las tres erres ecológicas: Reducir, reutilizar, reciclar. Obtenido de https://www.ifeelmaps.com/blog/2014/07/regla-de-las-tres-erres-ecologicas-reducir--reutilizar--reciclar

Martinez, M. (2014). EDUCACION EN LA BUSQUEDA DE UNA. Obtenido de http://repositorio.unicartagena.edu.co:8080/jspui/bitstream/11227/1150/1/Proyec to\%20Ambiental\%20Universitario.pdf

Mata, A., \& Gálvez, C. (2014). Reciclaje de vidrio. Obtenido de https://s3.amazonaws.com/academia.edu.documents/45578570/cal010.pdf?AWS AccessKeyId=AKIAIWOWYYGZ2Y53UL3A\&Expires=1539554894\&Signatur e=Lgs3cm1rNoxrYeU4oANf5k5qVQU\%3D\&response-contentdisposition=inline $\% 3 \mathrm{~B} \% 20$ filename\%3DRECICLAJE_DE_VIDRIO.pdf 
Medina, M. (2016). Manejo de desechos solidos y desarrollo sustentable. Obtenido de file:///C:/Users/LABIBLIOTECA/Downloads/1284Texto\%20del\%20art\%C3\%ADculo-4705-1-10-20161225.pdf

Melanv, V. (2014). Medio Ambiente. Obtenido de https://es.scribd.com/document/231850974/Medio-Ambiente

R. del Valle-Zermeño, J. G.-M.-P. (2017). Material characterization of the MSWI bottom ash as a function of particle size. Effects of glass recycling over time. Obtenido de https://www.sciencedirect.com/science/article/pii/S0048969717300426

Schmelzer, J. W., Fokin, V. M., \& Abyzov, A. S. (2016). Crystallization of Glass: What We Know, What We Need to Know. Obtenido de https://www.ingentaconnect.com/search/article?option1=tka\&value1=definition + of + glass \&pageSize $=10 \&$ index $=1 \#$ Data

Torrence, R. (2015). Ancient glass: from kaleidoscope to crystal ball. Obtenido de https://ac.els-cdn.com/S030544031500059X/1-s2.0-S030544031500059Xmain.pdf?_tid=8cb1d3fd-6ea5-4555-b6adc8328267ed9f\&acdnat=1539394018_179106fa5d72d3815eea5fc1d06e6927

Verger, E. (2017). ¿Cómo se fabrica el vidrio? ¿Qué tipos de vidrio existen? Obtenido de https://www.google.com.ec/search?q=BENEFICIOS+DEL+RECICLAJE\&oq= BENEFICIOS+DEL+RECICLAJE\&aqs=chrome..69i57j69i59j69i6012j012.2759 $4 \mathrm{j} 0 \mathrm{j} 7 \&$ sourceid $=$ chrome $\& \mathrm{ie}=\mathrm{UTF}-8$

Vigil, J. (2018). Reciclaje del vidrio. Obtenido de http://hogar.crisa.com/Acerca-de/Quees-el-Vidrio/Reciclaje-del-Vidrio

Vikinga. (2014). ARCHIVO DE LA ETIQUETA: ¿QUIENES RECICLAN MAS EN AMÉRICA LATINA? Obtenido de https://lamezcolanza.wordpress.com/tag/quienes-reciclan-mas-en-america-latina/ 


\section{Para citar el artículo indexado.}

Carrasco T. (2017). El reciclaje de vidrio y sus beneficios en el medio ambiente. Revista electrónica Explorador Digital 1(1), 17-27. Recuperado desde:

http://cienciadigital.org/revistacienciadigital2/index.php/exploradordigital/article/view/315/7 27

\section{Liencia}

El artículo que se publica es de exclusiva responsabilidad de los autores y no necesariamente reflejan el pensamiento de la Revista Explorador Digital.

El articulo queda en propiedad de la revista y, por tanto, su publicación parcial y/o total en otro medio tiene que ser autorizado por el director o editor de la Revista Explorador Digital.
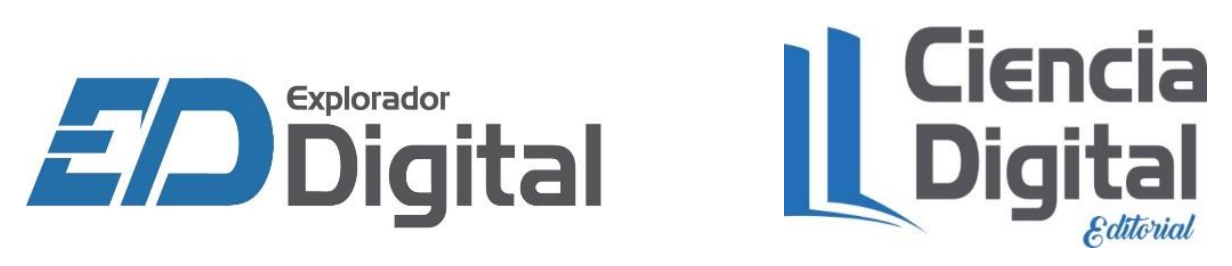